\title{
Weniger OFF-Phasen mit Opicapon
}

Fragestellung: Verringert Opicapon als Add-on zu L-Dopa die OFF-Zeiten beim fortgeschrittenen idiopathischen ParkinsonSyndrom (IPS)?

Hintergrund: Aktuell werden die COMT-Inhibitoren Entacapon und Tolcapon in der Behandlung von OFF-Phasen im fortgeschrittenen IPS-Stadium eingesetzt. Nachteil von Entacapon ist die Notwendigkeit der kombinierten Gabe mit L-Dopa. Tolcapon kann unabhängig von L-Dopa gegeben werden, erfordert aber regelmäßige Kontrollen der Leberwerte.

Patienten und Methodik: In der randomisierten, doppelblinden, multizentrischen Phase-III-Studie wurden 600 Patienten in fünf Gruppen (Opicapon $5 \mathrm{mg}, 25 \mathrm{mg}, 50 \mathrm{mg}$, Placebo und Entacapon in der Dosierung von $200 \mathrm{mg}$ als Add-on zu jeder L-Dopa-Gabe) verglichen. Dosisänderungen der L-Dopa-Begleitmedikation, nicht jedoch Änderungen der Einnahmefrequenz waren in den ersten drei Wochen nach Stu-

Ferreira JJ, Lees A, RochaJF et al. Opicapone as an adjunct to levodopa in patients with Parkinson's disease and end-ofdose motor fluctuations: a randomised, double-blind, controlled trial. Lancet Neurology 2016; 15: 154-65 dieneinschluss möglich. Die Studiendauer betrug 15 Wochen, der primäre Endpunkt war die Änderung der absoluten Zeit im OFF zwischen Baseline und Studienende. Sekundäre Endpunkte waren Änderungen im Anteil der
Patienten mit mehr als einer Stunde Reduktion der OFF-Zeit und eine Zunahme von mindestens einer Stunde ON-Zeit anhand von Patientenfragebögen [1] sowie skalenbasierte Änderungen.

Ergebnisse: 542 Patienten (90\%) beendeten die Studie. Die einmalige Einnahme von 50 mg Opicapon zur Nacht zeigte eine Überlegenheit gegenüber Placebo und eine Nichtunterlegenheit gegenüber Entacapon in der Reduktion der OFF-Zeit, die sich durchschnittlich um circa zwei Stunden pro Tag reduzierte (116,8 min). Dies entsprach im Vergleich zu Entacapon und Placebo einer Verbesserung von circa 30 beziehungsweise 60 Minuten. Die sekundären fragebogenbasierten Endpunkte im Vergleich zu Placebo wurden ebenfalls für $50 \mathrm{mg}$ Opicapon erreicht, unterschieden sich aber nicht von der Entacapongruppe. In den sekundären Endpunkten zeigten sich für den CGI-C (Clinician's Global Impression-Change Scale) und PGI-C (Patient's Global Impression-Change Scale) signifikante Änderungen im Vergleich zu Placebo sowie eine Überlegenheit gegenüber Entacapon. Unerwünschte Wirkungen waren insgesamt gering und nicht signifikant unterschiedlich zwischen den fünf Gruppen.

Schlussfolgerungen: Die einmal tägliche Einnahme von $50 \mathrm{mg}$ Opicapon reduzierte signifikant die OFF-Zeit, ohne dass vermehrt störende Dyskinesien auftraten. Opicapon könnte zu einer Verlängerung des Dosisintervalls und einer Verringerung der Zahl der L-Dopa-Einnahmezeitpunkte führen.

\section{- Kommentar von Franz Marxreiter und Jürgen Winkler, Erlangen}

\section{Einmalgabe von Opicapon - ein Vorteil?}

Aufbau und Durchführung der Studie erfüllen einen hohen methodischen Standard. Die einmal tägliche Einnahme von Opicapon, ohne Blutmonitoring, ist ein wichtiges Alleinstellungsmerkmal in der Gruppe der COMT-Inhibitoren und kann zu einem vereinfachten Therapieregime beitragen. In der Reduktion der OFF-Zeit liegt Opicapon (50 mg) zwischen Entacapon und Tolcapon [3]. Opicapon, aber auch Entacapon führten in dieser Studie im Vergleich zu Tolcapon [3] nur zu einer mäßigen Erniedrigung der L-Dopa-Tagesdosis, am wahrscheinlichsten weil Änderungen im L-Dopa-Regime nur in den ersten drei Wochen nach Einschluss möglich waren. Die Schlussfolgerung der Autoren bezüglich der Verlängerung des Dosisintervalls und der Verringerung der Zahl der L-Dopa-Einnahmezeitpunkte ist zwar nachvollziehbar, wurde in dieser Studie kritisch betrachtet aber nicht untersucht.

Die bei Einschluss bestehende mittlere OFF-Zeit von sechs Stunden und der mittlere UPDRS III waren ähnlich zu den Zulassungsstudien zu Entacapon [2] und Tolcapon [3] . Somit können die Ergebnisse dieser Studien gut miteinander verglichen werden. Da schwere OFF- Phasen ein Ausschlusskriterium in der vorliegenden Studie waren, bleibt die Interpretation der Ergebnisse hinsichtlich des Einsatzes von Opicapon insbeson- dere bei Patienten mit schweren Motorfluktuationen eingeschränkt. Absolut betrachtet, zeigte sich unter 50 mg Opicapon im Vergleich zu Entacapon eine Verdoppelung der Dyskinesien und eine Vervierfachung der Halluzinationen. Die Fallzahlen sind hier aber zu gering um daraus ein unterschiedliches Nebenwirkungsspektrum abzuleiten.
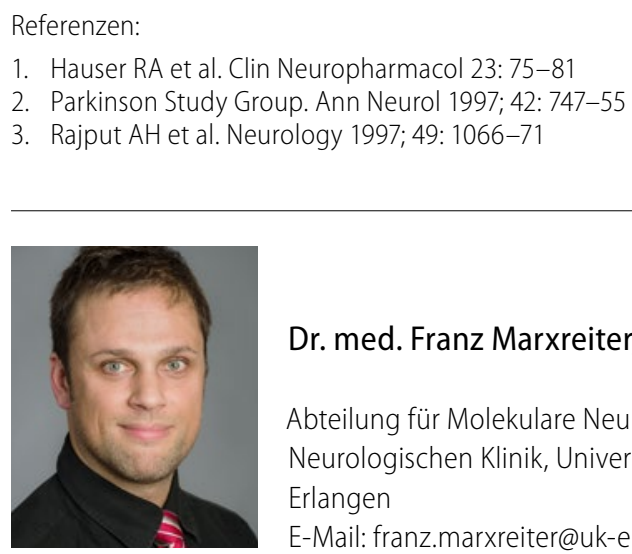

Dr. med. Franz Marxreiter, Erlangen

Abteilung für Molekulare Neurologie in der Neurologischen Klinik, Universitätsklinikum Erlangen

E-Mail:franz.marxreiter@uk-erlangen.de 